\title{
High-Resolution Neutron Diffraction Setting for Studies of Macro- and Microstrains in Polycrystalline Materials
}

\author{
Pavol Mikula ${ }^{1, a,{ }^{*}}$, Massimo Rogante ${ }^{2, b}, J^{2}$ Saroun ${ }^{1, c}$ and Miroslav Vrána ${ }^{1, d}$ \\ ${ }^{1}$ Nuclear Physics Institute ASCR, v.v.i., Husinec-Řež 130, 25068 Řež, Czech Republic \\ ${ }^{2}$ Rogante Engineering Office, I-62012 Civitanova Marche, Italy \\ amikula@ujf.cas.cz, ㅁmain@roganteengineering.it, 'saroun@ ujf.cas.cz, dvrana@ ujf.cas.cz
}

Keywords: Neutron Diffraction, Bent Perfect Crystals, Bragg Diffraction Optics, Polycrystals, Strains

\begin{abstract}
On the basis of our previous experience [1-4], a unique three axis high-resolution experimental setting for nondestructive strain measurements which is based on neutron Bragg diffraction optics with cylindrically bent perfect crystals is presented. The use of focusing in real and namely, in momentum space, from FWHM of diffraction lines the three axis setting provides the $\Delta d / d$ resolution ( $d$-lattice spacing) of about $4 \times 10^{-3}$ for bulk samples. It permits studies not only macrostrain components resulting from angular shifts of diffraction peaks but also estimations of microstrains in a plastically deformation region by means of profile-broadening analysis. The feasibility of the experimental setting is demonstrated on low carbon steel shear deformed steel wires.
\end{abstract}

\section{Introduction}

Non-destructive X-ray and neutron diffraction techniques for studies of internal strain fields in polycrystalline materials have been successfully used for many years [5-17]. At present, the investigations of residual strains/stresses are usually carried out at the dedicated double axis diffractometers (strain scanners) with a bent perfect crystal (BPC) focusing monochromator situated on the first axis, a sample situated on the second axis and with a position sensitive detector (PSD). With respect to the experimental conditions, the BPC crystal is optimally bent which results in a highly collimated beam (often called quasiparallel beam) reflected by the polycrystalline sample [12,15-17]. However, the $\Delta d / d$ resolution of these dedicated scanners derived from the FWHM of the diffraction lines is sufficiently high for small sample gauge volumes but rarely better than $8 \times 10^{-3}$ for bulk samples. Important thing is that the dedicated scanners operate with open beams without the necessity of Soller collimators. A further way, how to increase the resolution which would permit to investigate an influence of microstrains on the diffraction profile, namely, in the case of plastically deformed samples, is the use of a three axis set-up when employing a third BPC crystal on the third axis as an analyzer. The first attempts of the use of the three axis set-up were described two decades ago [1,2]. The drawback of such a set-up in comparison with the conventional scanners consists in using the step-by-step analysis (by rocking the BPC analyzer). Therefore, the effective measurements could be carried out within a reasonable measurement time at high-flux neutron sources. From the point of view of luminosity, some improvements can be done by an employment of the BPC monochromator at a rather small Bragg angle, while the resolution can be optimized by a suitable choice of the BPC analyzer and its thickness, as it was used in the present case (see Fig. 1). 


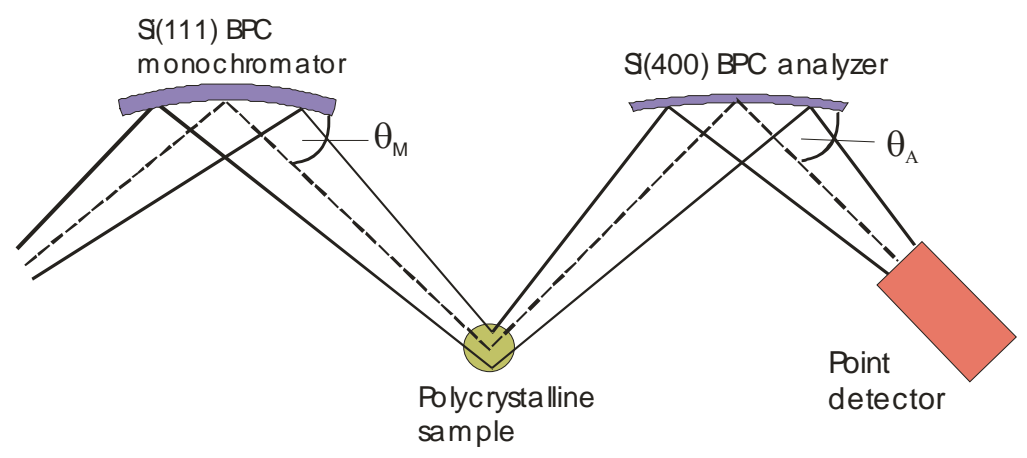

Fig. 1. Schematic diagram of the experimental performance with the sample in the vertical position.

\section{Experimental details}

The set-up shown in Fig. 1 was experimentally realized on the three axis neutron optic diffractometer installed at the medium power research reactor LVR-15 situated in Řež. Si(111) and $\mathrm{Si}(400)$ single crystals had the dimensions of $200 \times 40 \times 4 \mathrm{~mm}^{3}$ and $20 \times 40 \times 1.3 \mathrm{~mm}^{3}$ (length $\mathrm{x}$ width $\mathrm{x}$ thickness), respectively. The monochromator $\mathrm{Si}(111)$ had a fixed curvature with a radius $R_{\mathrm{M}}$ of about $12 \mathrm{~m}$. The curvature of the analyzer $\mathrm{Si}(400)$ was changeable in the range from $R_{\mathrm{M}}=36 \mathrm{~m}$ to $R_{\mathrm{M}}=3.6 \mathrm{~m}$ and finally set for the optimum radius of curvature of $R_{\mathrm{A}}=9 \mathrm{~m}$, where a best resolution was found. For a practical demonstration of the feasibility of using the three axis set-up for diffraction line analysis we used $\alpha$-Fe(110) nondeformed as well as deformed wires with accumulated shear deformation, as a result of rolling with the shear of the metal ingot and conventional wire drawing. Due to the deformation the diameters of the samples are not the same, however, but they are in the vicinity of $5 \mathrm{~mm}$. The samples were already studied in detail elsewhere [18] and in this case several of them were used for the feasibility studies of the set-up. The description of the nondeformed as well as with accumulated shear deformation samples (low carbon steel - Grade 08G2S GOST 1050) is shown in Table1. Table 2 describes the chemical composition of the steel.

Table 1. Description of deformation of the low-alloyed steel samples. Percentage in the fourth column shows the reduction degree in drawing deformation.

\begin{tabular}{c|c|c|c|}
\hline $\begin{array}{c}\text { Sample } \\
\text { number }\end{array}$ & $\phi[\mathrm{mm}]$ & $\begin{array}{c}\text { Shear def. } \\
{[\%]}\end{array}$ & $\begin{array}{c}\text { Drawing def. } \\
{[\%]}\end{array}$ \\
\hline 1 & 5.10 & 0 & 0 \\
\hline 2 & 4.28 & 8 & 23.2 \\
\hline 3 & 5.35 & 0 & 0 \\
\hline 4 & 4.28 & 16.6 & 23.2 \\
\hline 5 & 5.57 & 0 & 0 \\
\hline 6 & 4.28 & 23 & 23.2 \\
\hline
\end{tabular}


Table 2. Chemical composition of low-alloyed structural steel grade 08G2S GOST 1050 element

$\begin{array}{cccccccccc}\text { Element } & \mathbf{C} & \mathbf{M n} & \mathbf{S i} & \mathbf{S} & \mathbf{P} & \mathbf{C r} & \mathbf{N i} & \mathbf{C u} & \mathbf{N 2} \\ \text { wt \% } & 0,08 & 1,87 & 0,82 & 0,020 & 0,022 & 0,02 & 0,02 & 0,02 & 0,007\end{array}$

\section{Experimental results of the diffraction profiles - Radial components}

The steel samples were situated on the second axis of the diffractometer in vertical position. The width of the incident beam was $8 \mathrm{~mm}$ and therefore, the whole volume of the sample was irradiated (within the whole diameter). The height of the incident beam was $20 \mathrm{~mm}$. From the introduced Fig. 2, we can detect the following features: The peak intensities and FWHMs related to the deformed samples differ very little. This is brought about by the fact that the diameter of the deformed samples was equal and that the shear deformation has a negligible effect. Very close values of $F W H M$ point out on the fact that the lattice deformation in the radial direction was basically brought about by drawing deformation. The integrated intensity under the peak profiles related to the nondeformed samples N.1, N.3 and N.5 primarily corresponds to the irradiated volume of the sample, which is naturally maximum for the sample N. 5.
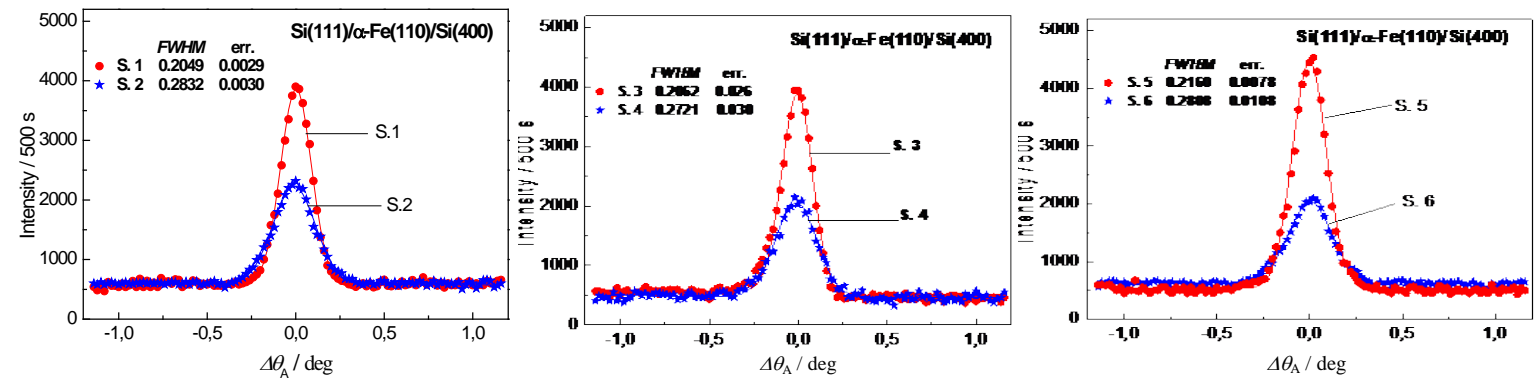

Fig. 2. Diffraction profiles related to the samples N.1-N.6 situated in the vertical position as analyzed by the bent perfect $\mathrm{Si}(400)$ analyzer.

\section{Experimental results of the diffraction profiles - Axial components}

In the next step the steel samples were situated on the second axis of the diffractometer in the horizontal position. The obtained results are shown in Fig. 3. In comparison with the previous case, for the samples in the horizontal position their irradiated volume is much smaller and
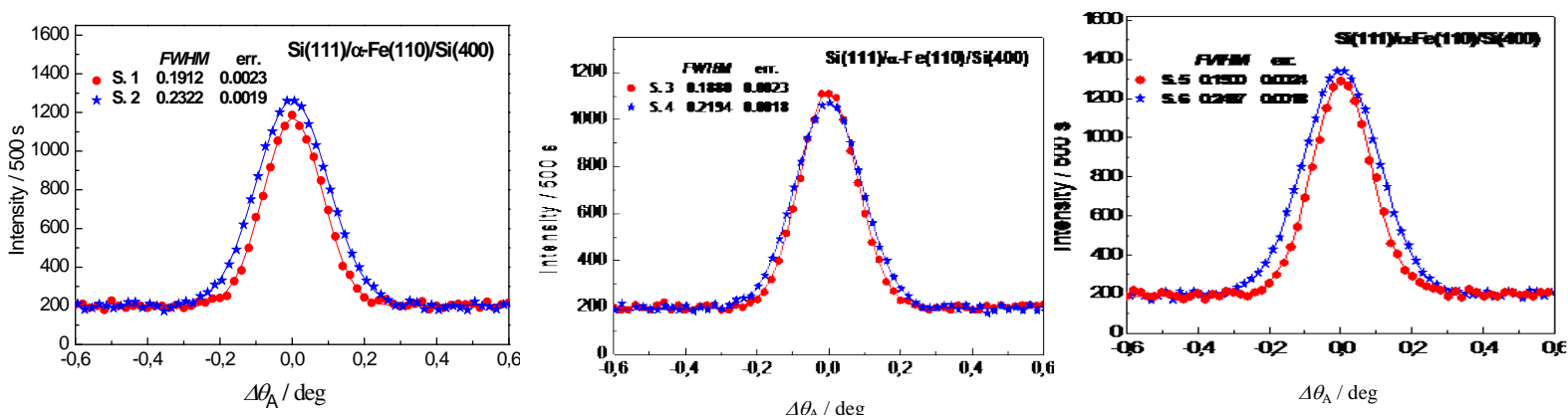

Fig. 3. Diffraction profiles related to the samples N.1-N.6 situated in the horizontal position for measurement of the axial component as analyzed by the bent perfect Si(400) analyzer.

correspondingly neutron signal was smaller. In this case, it was found that the resolution was dependent on the width of the incident beam impinging the sample and therefore, we used the slit width of $5 \mathrm{~mm}$. It can be seen from Fig. 3 that the resolution represented by FWHM was practically the same for all nondeformed samples N.1, N.3 and N.5. Small differences in FWHM 
can be seen for plastically deformed samples. It points out the fact that the lattice deformation was basically brought about by drawing deformation and influencing the radial strain component and much less the axial strain component.

\section{Summary}

The presented neutron diffraction results obtained on the samples of low-alloyed quality structural steel (Grade 08G2S GOST 1050) document the feasibility of the unconventional three axis set-up for studies of some properties of polycrystalline samples in the plastic deformation region. It can be seen from the Tab. 3 that though the FWHM-effects resulting from the applied

Table 3. Summary of the FWHMs as calculated in ( $\Delta \mathrm{d} / \mathrm{d})$ - scale

\begin{tabular}{|c|c|c|c|c|c|c|}
\hline Sample number & N.1 & N.2 & N.3 & N.4 & N.5 & N.6 \\
\hline$F W H M(\Delta d / d)$ Radial $\left[10^{-3}\right]$ & 4.09 & 5.66 & 4.12 & 5.44 & 4.32 & 5.61 \\
\hline$F W H M(\Delta d / d)$ Axial $\left[10^{-3}\right]$ & 3.82 & 4.64 & 3.76 & 4.38 & 3.80 & 4.97 \\
\hline
\end{tabular}

deformation on the samples are very small, thanks to the high resolution of the experimental setup, they are clearly measurable. In particular, such results can be used as an additional support to complement the information achieved by using the other characterization methodologies. It should be pointed out that contrary to the conventional double axis strain scanner, the three axis setting provides a high resolution for bulk samples e.g. of the diameter of 5-10 mm (for the radial component) while for conventional scanner such diameter itself introduces to the resolution an uncertainty in FWHM of the diffraction profile at least of $1 \times 10^{-2}$ rad. Thanks to the high resolution property of the three axis set-up, it can be, of course, used also for measurements of residual elastic deformation macrostresses, however, less efficiently in comparison with the conventional two axis neutron diffraction measurement [18] which has not so high resolution requirements.

\section{Acknowledgement}

Measurements were carried out at the CANAM infrastructure of the NPI CAS, v.v.i. in Řzž supported through MŠMT project No. LM2015056. The presented results were also supported in the frame of LM2015074 infrastructural MŠMT project "Experimental nuclear reactors LVR-15 and LR-0" as well as by the Czech Science Foundation GACR through the project No. 1608803J. The authors would like to thank Ms. Michalcová for the help in measurements and evaluations of the data.

\section{References}

[1] J. Kulda, P. Mikula, P. Lukáš and M. Kocsis, Utilisation of bent Si crystals for elastic strain measurements, Physica B 180\&181 (1992) 1041-1043.

[2] M. Vrána, P. Lukáš, P. Mikula, and J. Kulda, Bragg diffraction optics in high resolution strain measurements, Nucl. Instrum. Methods in Phys. Research A, 338 (1994) 125-131. https://doi.org/10.1016/0168-9002(94)90172-4

[3] B.S. Seong, V. Em, P. Mikula, J. Šaroun, M.H. Kang, Unconventional Performance of a Highly Luminous Strain/Stress Scanner for High Resolution Studies, Materials Science Forum, 681 (2011) 426-430. https://doi.org/10.4028/www.scientific.net/MSF.681.426 
[4] P. Mikula, M. Vrána, J. Šaroun, B.S. Seong, W. Woo, Double Bent Crystal Monochromator for High Resolution Neutron Powder Diffraction, Powder Diffraction, 28, Issue S2 (2013) S351S359. https://doi.org/10.1017/S0885715613000912

[5] I.C. Noyan and J.B. Cohen, Residual stress: measurement by diffraction and interpretation, 1st edn; 1987, New York, Springer-Verlag. https:/doi.org/10.1007/978-1-4613-9570-6

[6] M.T. Hutchings, and A.D. Krawitz, (Eds.), 1992, "Measurement of residual and applied stress using neutron diffraction", NATO ASI Series, Applied Sciences, Vol. 26, Kluwer Academic Publisher, 1992.

[7] M. R. Daymond, M. A. M. Bourke, R. B. von Dreele, B. Clausen, and T. Lorentzen, Use of Rietveld refinement for elastic macrostrain determination and for evaluation of plastic strain history from diffraction spectra, J. Appl. Phys. 82 (1997) 1554-1562.

https://doi.org/10.1063/1.365956

[8] G.A. Webster (ed.), Polycrystalline materials-determination of residual stress by neutron diffraction, ISO/TTA3, ISO/Technology, Trends Assessment, Geneva, Switzerland, 2001.

[9] V. Stelmukh, L. Edwards, J.R. Santisteban, S. Ganguly, and M.E. Fitzpatrick, Weld stress mapping using neutron and synchrotron x-ray diffraction, Materials Science Forum, 404-407 (2002) 599-604. https://doi.org/10.4028/www.scientific.net/MSF.404-407.599

[10] M.T. Hutchings, P.J. Withers, T.M. Holden and T. Lorentzen, Introduction to the characterization of residual stress by neutron diffraction, 1st ed.; 2005, London, Taylor and Francis.

[11] P. Mikula, M. Vrána and P. Lukáš, Power of Bragg Diffraction Optics for High Resolution Neutron Diffractometers for Strain/Stress Scanning, In IAEA-TECDOC-1457 "Measurement of residual stress in materials using neutrons”, IAEA Vienna, 2005, pp. 19-27.

[12] T. Pirling, G. Bruno, and P.J. Withers, SALSA, Advances in Residual Stress Measu-rement at ILL, Materials Science Forum, 524-525 (2006) 217-22.

https://doi.org/10.4028/www.scientific.net/MSF.524-525.217

[13] T.M. Holden, H. Suzuki, D.G. Carr, M.I. Ripley and B. Clausen, Stress measurements in welds: Problem areas, Mater. Sci. Eng. A, 437 (2006) 33-37.

https://doi.org/10.1016/j.msea.2006.04.055

[14] P. J. Withers, Comptes Rendus Physique, Mapping residual and internal stress in materials by neutron diffraction, 8 (2007) 806-820.

[15] P. Mikula, M. Vrána, L. Mráz and L. Karlsson, High-Resolution Neutron Diffraction Employing Bragg Diffraction Optics - A Tool for Advanced Nondestructive Testing of Materials, In Proc. of ASME Conference on Engineering Systems Design and Analysis, 7 to 9 July 2008, Haifa. ISBN 0-7918-3827-7. https://doi.org/10.1115/ESDA2008-59174

[16] R.C. Wimpory, P. Mikula, J. Šaroun, T. Poeste, Junghong Li, M. Hoffman nand R. Schneider, Efficiency Boost of the Materials Science Diffractometer E3 at BENSC: One Order of Magnitude Due to a Double Focusing Monochromator, Neutron News, 19 (2008) 16-19. https://doi.org/10.1080/10448630701831995 
[17] W. Woo, Z. Feng, X. Wang and S.A. David, Neutron diffraction measurements of residual stresses in friction stir welding: A review, Science and Technology of Welding and Joining, 16 (2011) N.1 23-32.

[18] M. Rogante, P. Mikula, P. Strunz, A. Zavdoveev, Residual stress determination by neutron diffraction in low-carbon steel wires with accumulated shear deformation, Proc. 7th Int. Conf. "Mechanical Technologies and Structural Materials" MTMS2017, Split, Croatia, 21-22 Sept. 2017, S. Jozić, B. Lela, Eds., Croatian Society for Mechanical Technologies, Split, Croatia (2017), pp. 111-115. 